\title{
Atención farmacéutica domiciliaria a pacientes frágiles en Gipuzkoa
}

\section{Xabier Aizpurua-Arruti ${ }^{1,2}$, Amaia Malet-Larrea', Ainhoa Oñatibia-Astibia', Ana del Pozo-Rodríguez², Estibaliz Goyenechea', Miguel Ángel Gastelurrutia ${ }^{1,3}$}

1. Colegio Oficial de Farmacéuticos de Gipuzkoa, Donostia/San Sebastián. 2. Facultad de Farmacia (Universidad del País Vasco). Grupo de Farmacocinética, Nanotecnología y Terapia génica (PharmaNanoGene), Vitoria-Gasteiz. 3. Grupo de Investigación de Atención Farmacéutica, Universidad de Granada.

\section{PALABRAS CLAVE}

Atención Farmacéutica Domiciliaria, Atención Farmacéutica, Servicios Profesionales Farmacéuticos Asistenciales

\section{ABREVIATURAS}

AFD: Atención Farmacéutica Domiciliaria

COFG: Colegio Oficial de Farmacéuticos de Gipuzkoa PRM: Problemas Relacionados con la Medicación

RNM: Resultados Negativos asociados a la Medicación

SFT: Seguimiento

Farmacoterapéutico

SPFA: Servicios Profesionales

Farmacéuticos Asistenciales

\section{KEYWORDS}

Home Pharmaceutical Care, Pharmaceutical Care, Professional Pharmaceutical Care Services

\section{RESUMEN}

Introducción:la asistencia sanitaria a domicilio es una de las estrategias que impulsan las administraciones para pacientes pluripatológicos que viven en sus domicilios y que debido a su grado de discapacidad o vulnerabilidad no pueden desplazarse a los centros sanitarios. Este estudio consiste en pilotar la Atención Farmacéutica Domiciliaria (AFD) ofreciendo respuestas individualizadas a los pacientes en sus domicilios, dentro del equipo multidisciplinar de atención a los pacientes frágiles de la Fundación Hurkoa.

Material y métodos: se convocó a todas las farmacias de los municipios de Irún y Azkoitia a una formación. Los pacientes incluidos en el Plan Integral de Atención a la Fragilidad de la Fundación Hurkoa, que requerían ayuda con la gestión de la medicación y aceptaron incluir a un farmacéutico en el equipo multidisciplinar de cuidados, seleccionaron su farmacia comunitaria. Los farmacéuticos de dichas farmacias realizaron una revisión del botiquín junto con la revisión de la medicación.

Resultados: en la revisión del botiquín se detectaron medicamentos caducados o no utilizados y se retiraron entre 2 y 3 medicamentos a todos los pacientes. En la revisión de la medicación se detectaron al menos un Problema Relacionado con la Medicación (PRM) en todos los pacientes del estudio. En la evaluación de la satisfacción el $100 \%$ de los pacientes refirieron un grado de satisfacción alto con el programa.

Discusión: el presente estudio refuerza la importancia de la inclusión del farmacéutico comunitario en el equipo multidisciplinar de AFD, ofreciendo respuestas individualizadas a cada paciente, mejorando la gestión de su medicación y así consiguiendo mejoras significativas en la calidad de vida.

\section{Home pharmaceutical care for frailty patients in Gipuzkoa}

\section{ABSTRACT}

Introduction: Home health care is one of the strategies promoted by administrations for patients with multiple pathologies, who live at home and who, due to their degree of disability or vulnerability, cannot go to health centers. This study consists of piloting home pharmaceutical care (HFC) by offering individualized responses to patients at home, within the multidisciplinary team for the care of frailty patients of the Hurkoa Foundation.

Material and methods: All pharmacies in the municipalities of Irún and Azkoitia were summoned to a training session. Patients included in the Hurkoa Foundation's Comprehensive Fragility Care Plan who required help with medication management and agreed to include a pharmacist in the multidisciplinary care team, selected their community pharmacy. Pharmacists at these pharmacies conducted a review of the medicine cabinet (brown bag) along with the medication review.

Results: In the review of the medicine cabinet (brown bag), expired or unused medications were detected and between 2 and 3 medications were withdrawn from all patients. In the review of the medication, they detected at least one "PRM" in all the patients in the study. In the satisfaction evaluation, $100 \%$ of the patients reported a high degree of satisfaction with the program.

Discussion: The present study reinforces the importance of including the community pharmacist in the HFC multidisciplinary team, offering individualized responses to each patient, improving the management of their medication and thus achieving significant improvements in quality of life.
Recibido: 04/01/2021

Aceptado: 05/04/2021

Disponible online: $02 / 07 / 2021$
Financiación: ninguna.

Conflicto de intereses: ninguno.

Cite este artículo como: Aizpurua-Arruti X, Malet-Larrea A, Oñatibia-Astibia A, del-Pozo-Rodríguez A, Goyenechea E, Gastelurrutia MA. Atención farmacéutica domiciliaria a pacientes frágiles en Gipuzkoa. Farmacéuticos Comunitarios. 2021 Jul 02; 13 (3): 17-24. doi:10.33620/FC.2173-9218.(2021/Vol13).003.03

Correspondencia: Xabier Aizpurua (xabieraizpurua@cofgipuzkoa.eus).

ISSN 1885-8619 @SEFAC (Sociedad Española de Farmacia Clínica, Familiar y Comunitaria). Todos los derechos reservados. 


\section{INTRODUCCIÓN}

La disminución de la mortalidad en la edad avanzada ha prolongado la esperanza de vida de la población, aumentando el número de personas con enfermedades crónicas, frágiles, vulnerables y/o dependientes $(1,2)$.

La necesidad de una continuidad asistencial en la atención al paciente crónico y pluripatológico, en todos los estadios de su enfermedad e independientemente de su dependencia, ha quedado ampliamente avalada en los últimos años (3), incluyendo la asistencia farmacéutica dentro del equipo multidisciplinar (4).

La asistencia sanitaria a domicilio es una de las estrategias que impulsan las administraciones sanitarias para pacientes pluripatológicos, no ingresados en centros asistenciales, que viven en sus domicilios y que, debido a su grado de discapacidad o vulnerabilidad, no pueden desplazarse a los centros sanitarios $u$ hospitales (3).

La Atención Farmacéutica Domiciliaria (AFD) se define como la prestación de Servicios Profesionales Farmacéuticos Asistenciales (SPFA) en el domicilio a aquellos pacientes que por su condición sanitaria no pueden acudir a la farmacia comunitaria para recibirlos (5).

A nivel internacional hay varias experiencias en AFD. En Australia, Canadá y Estados Unidos se han desarrollado estudios que han demostrado la eficacia y la necesidad de prestar el servicio de AFD por parte de un equipo multidisciplinar, entre los que se encuentra el farmacéutico comunitario (6-9). En España, sin embargo, este concepto es relativamente reciente y constituye un nuevo reto para la profesión.

Una experiencia relevante en el campo de la AFD es la del programa Domi-EQlfar, llevado a cabo en la Comunidad Valenciana. Se trata de un programa de AFD dirigido a pacientes dependientes con cuidadores no profesionales (pacientes no institucionalizados) con el objetivo de ofrecer un servicio de atención farmacéutica integral, que garantizó la equidad, la calidad y la integración. En dicho programa, se observó que hubo una mejora en la comunicación entre profesionales y en los resultados en salud de los pacientes beneficiados (10).

En este contexto, el Colegio Oficial de Farmacéuticos de Gipuzkoa (COFG), en colaboración con la Fundación Hurkoa (organización sin ánimo de lucro para atender y defender los derechos de personas mayores -dependientes, en soledad y aislamiento- o con enfermedad mental en situación de fragilidad), diseñó un programa piloto de AFD dirigido a pacientes frágiles dependientes, pertenecientes todos ellos al Plan Integral de Atención a la Fragilidad de la Fundación (11). El objetivo principal de este estudio consiste en pilotar la AFD por parte de los farmacéuticos comunitarios de Irún y Azkoitia ofreciendo respuestas individualizadas a las necesidades de los pacientes en sus domicilios, dentro del equipo multidisciplinar de atención a los pacientes frágiles de la Fundación Hurkoa.

\section{MATERIAL Y MÉTODOS}

La integración de la AFD en el cuidado integral del paciente frágil dentro del programa de la Fundación Hurkoa comenzó en el año 2016 en Irún, ampliándose a la localidad de Azkoitia en 2017.

\section{Formación a farmacias}

El COFG convocó a todas las farmacias comunitarias de los municipios de Irún y Azkoitia a una formación en la que se trataron los siguientes aspectos: Fundación Hurkoa y su Plan Integral de Atención a la Fragilidad, objetivos de la integración de la farmacia en el Programa, procedimiento, servicios a realizar (revisión del botiquín, revisión de la medicación y seguimiento farmacoterapéutico, SFT), cronograma y registros.

\section{Selección de pacientes y farmacias}

El personal técnico de Hurkoa incluyó en su Plan Integral de Atención a la Fragilidad a las personas que cumplian los criterios para el mismo (11). Hurkoa elaboró el Plan Integral de Atención a la Fragilidad, en el que se valoraron las necesidades de los pacientes de forma individualizada. A quienes requerían ayuda con la gestión de la medicación se les ofreció la opción de incluir a un farmacéutico en el equipo multidisciplinar de cuidados. Las personas que aceptaron seleccionaron su farmacia comunitaria de referencia y se lo comunicaron al coordinador de Hurkoa. Este trasladó al coordinador del COFG por correo electrónico las iniciales del paciente y la farmacia seleccionada.

\section{Procedimiento}

El coordinador del COFG contactó por teléfono con las farmacias seleccionadas para trasmitirles la inclusión de cada nuevo paciente en el programa y solicitar el nombre y apellidos del farmacéutico responsable del caso. Se revisó el procedimiento del programa, la intervención farmacéutica y los registros y se facilitó al farmacéutico responsable del caso el correo electrónico y teléfono del coordinador de Hurkoa, para que acordasen y realizasen de forma conjunta la visita al domicilio para la provisión de la AFD.

\section{Servicio de Atención Farmacéutica Domiciliaria}

En la primera visita al domicilio, el farmacéutico responsable entrevistó al paciente o a la persona responsable de la medicación (cuidador, auxiliar domiciliario...) para que valorase la situación en referencia a la medicación y analizase todos los medicamentos que utilizaba, así como la posología y pautas de dosificación.

En primer lugar, el farmacéutico realizaba una revisión del botiquín junto con la revisión de la medicación. Durante la revisión, el farmacéutico debía detectar incidencias, como Problemas Relacionados con la Medicación (PRM). Si además de PRM detectaba posibles Resultados Negativos asociados a la Medicación (RNM), el siguiente servicio a proponer sería el SFT (figura 1). 


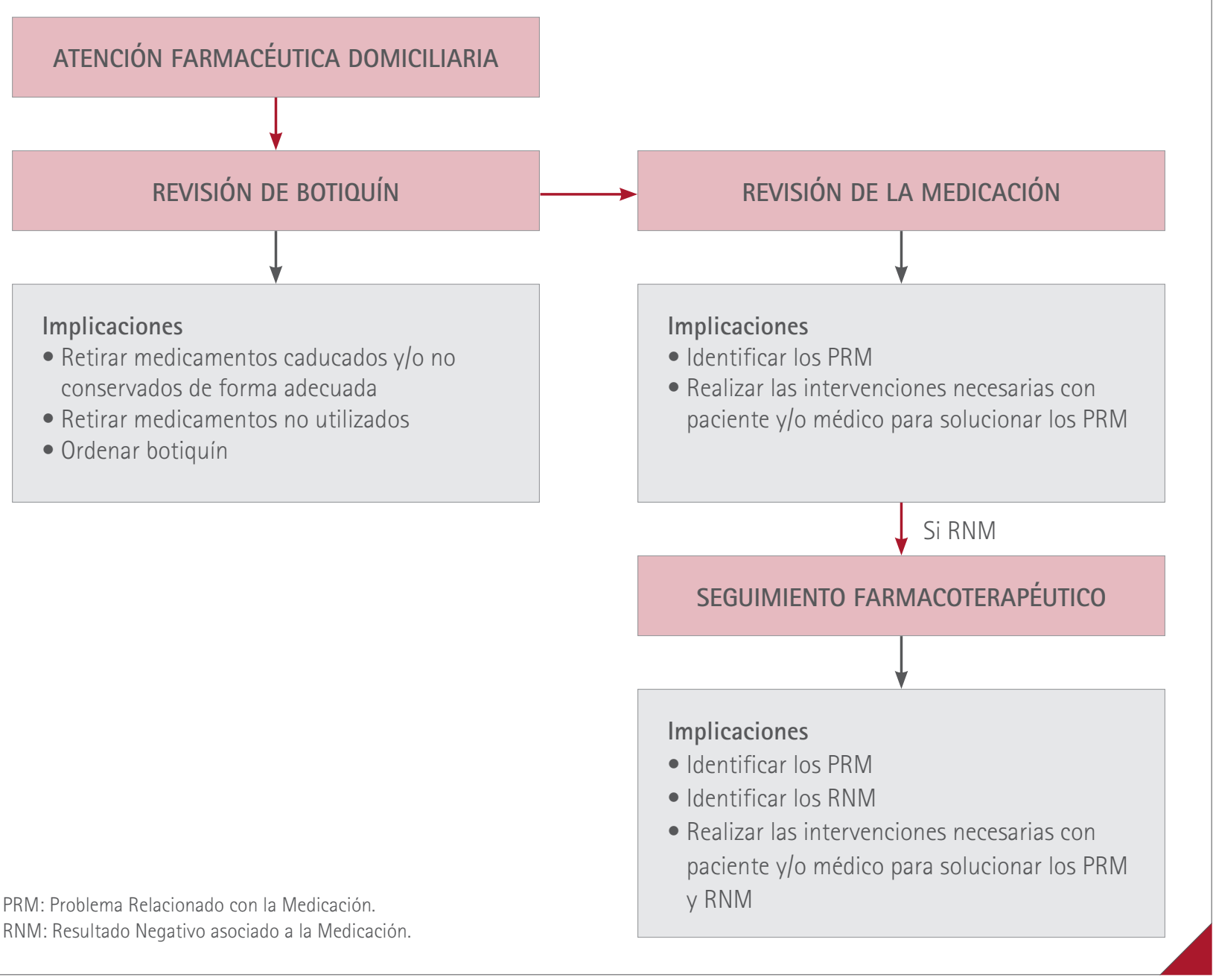

Figura 1 Algoritmo de la atención farmacéutica domiciliaria para el paciente frágil

Revisión del botiquín

El objetivo principal era retirar los medicamentos caducados, en malas condiciones de conservación o que ya no se utilizaban.

El farmacéutico utilizaba la hoja de tratamiento activo como guía para discernir aquellos medicamentos prescritos de los que no lo estaban. Siguiendo el orden de la hoja de tratamiento activo, se seleccionaban entre todos los medicamentos del botiquín aquellos incluidos en la hoja de tratamiento activo poniéndolos en un grupo aparte. Cada vez que se cogía un medicamento se comprobaba la fecha de caducidad y condiciones de conservación; en caso de que alguno no estuviese correcto, se separaba en el "grupo de medicamentos con incidencias".

Se preguntaba al paciente o a la persona responsable de la medicación cuáles de los medicamentos presentes en el botiquín no incluidos en la hoja de tratamiento activo utilizaba, de forma continua o a demanda. Se comprobaba la caducidad, condiciones de conservación y duplicidades con los medicamentos incluidos en la hoja de tratamiento activo. En caso de discrepancias, se separaba incluyéndolo en el "grupo de medicamentos con incidencias".
El farmacéutico argumentaba la razón por la que se debian desechar los medicamentos no utilizados y los introducía en una bolsa para depositarlos en el Punto SIGRE de la farmacia.

Finalmente, el farmacéutico introducía los medicamentos utilizados en el botiquín siguiendo en cada caso la estrategia más adecuada para su correcta clasificación (medicamentos crónicos vs. agudos; por problema de salud a tratar; por tomas a lo largo del día; etc.).

\section{Revisión de la medicación}

El objetivo principal era identificar y resolver cualquier posible PRM, así como solventar las posibles dudas del paciente o cuidador respecto a la medicación.

Para cada uno de los medicamentos que tomaba el paciente, tanto los incluidos en la hoja de tratamiento activo como los medicamentos sin receta, se valoraban los posibles PRM siguiendo un esquema basado en el Método Dáder (12) e indicado en la tabla 1. Algunos de los PRM se detectaban preguntando al paciente y otros por valoración del propio farmacéutico. El farmacéutico realizaba la intervención correspondiente, con el médico y/o paciente, para resolver cada PRM (tabla 1). 
Tabla 1 Procedimiento para la revisión de la medicación

\begin{tabular}{|c|c|c|c|c|}
\hline \multirow{2}{*}{\multicolumn{2}{|c|}{ Método de detección del PRM }} & \multirow{3}{*}{$\begin{array}{l}\text { Listado PRM } \\
\text { Dosis, pauta } \\
\text { y/o duración no } \\
\text { adecuada }\end{array}$} & \multicolumn{2}{|l|}{ Intervención } \\
\hline & & & \multirow{2}{*}{$\begin{array}{l}\text { Con paciente } \\
\text { Corregir dosis, pauta y/o } \\
\text { duración }\end{array}$} & \multirow{2}{*}{$\begin{array}{l}\text { Con médico } \\
\text { Informar con el objetivo de } \\
\text { modificar dosis, pauta y/o } \\
\text { duración }\end{array}$} \\
\hline \multirow{4}{*}{$\begin{array}{l}\text { Pregunta al } \\
\text { paciente }\end{array}$} & $\begin{array}{l}\text { ¿Cómo toma el } \\
\text { medicamento? } \\
\text { ¿Cuánto y cuándo lo toma? } \\
\text { ¿Cómo le va? ¿Nota el } \\
\text { efecto? }\end{array}$ & & & \\
\hline & $\begin{array}{l}\text { ¿Cómo utiliza el } \\
\text { medicamento? ¿Tiene } \\
\text { alguna dificultad en la } \\
\text { administración? ¿Conoce } \\
\text { alguna precaución de uso? }\end{array}$ & $\begin{array}{l}\text { Administración } \\
\text { errónea }\end{array}$ & $\begin{array}{l}\text { Corregir forma de } \\
\text { administración } \\
\text { Especial atención en } \\
\text { medicamentos complejos }\end{array}$ & \\
\hline & $\begin{array}{l}\text { ¿Se olvida alguna vez de } \\
\text { tomarlo? Si se encuentra } \\
\text { bien, ¿deja de tomarlo } \\
\text { alguna vez? y ¿si le sienta } \\
\text { mal? }\end{array}$ & Falta de adherencia & $\begin{array}{l}\text { Investigar causa de no } \\
\text { adherencia: } \\
\text { A) No intencionada: SPD y } \\
\text { supervisión periódica } \\
\text { B) Intencionada: intervenir } \\
\text { sobre la causa (eliminar } \\
\text { miedo a efectos adversos, } \\
\text { explicar importancia de } \\
\text { la medicación para el } \\
\text { problema de salud...) }\end{array}$ & $\begin{array}{l}\text { Si la causa de no } \\
\text { adherencia es } \\
\text { intencionada: } \\
\text { informar al médico } \\
\text { para valorar sustituir } \\
\text { medicamento }\end{array}$ \\
\hline & $\begin{array}{l}\text { ¿Nota algo extraño } \\
\text { relacionado con el } \\
\text { medicamento? }\end{array}$ & $\begin{array}{l}\text { Probabilidad de } \\
\text { efectos adversos }\end{array}$ & & $\begin{array}{l}\text { Informar con objetivo de } \\
\text { sustituir medicamento }\end{array}$ \\
\hline \multirow{7}{*}{\multicolumn{2}{|c|}{ Valoración del farmacéutico }} & Contraindicación & & $\begin{array}{l}\text { Informar con el objetivo de } \\
\text { sustituir el medicamento }\end{array}$ \\
\hline & & $\begin{array}{l}\text { Errores en la } \\
\text { dispensación }\end{array}$ & Corregir dispensación & \\
\hline & & $\begin{array}{l}\text { Errores en la } \\
\text { prescripción }\end{array}$ & & $\begin{array}{l}\text { Informar para solucionar } \\
\text { error }\end{array}$ \\
\hline & & Interacciones & $\begin{array}{l}\text { Consejos para evitar } \\
\text { interacción (distanciar } \\
\text { toma, alimentos...) }\end{array}$ & $\begin{array}{l}\text { Informar con el objetivo de } \\
\text { sustituir medicamento }\end{array}$ \\
\hline & & $\begin{array}{l}\text { Medicamento no } \\
\text { necesario }\end{array}$ & & $\begin{array}{l}\text { Informar con objetivo de } \\
\text { eliminar prescripción }\end{array}$ \\
\hline & & $\begin{array}{l}\text { Otros problemas de } \\
\text { salud que afectan al } \\
\text { tratamiento }\end{array}$ & $\begin{array}{l}\text { Consejos higiénico- } \\
\text { dietéticos y hábitos de vida } \\
\text { saludables }\end{array}$ & $\begin{array}{l}\text { Informar con objetivo de } \\
\text { modificar dosis / fármaco }\end{array}$ \\
\hline & & $\begin{array}{l}\text { Problema de salud } \\
\text { insuficientemente } \\
\text { tratado }\end{array}$ & $\begin{array}{l}\text { Consejos higiénico- } \\
\text { dietéticos y hábitos de vida } \\
\text { saludables }\end{array}$ & $\begin{array}{l}\text { Informar con objetivo de } \\
\text { instaurar otro fármaco }\end{array}$ \\
\hline \multirow{2}{*}{\multicolumn{2}{|c|}{ Detectados en revisión de botiquín }} & Duplicidad & $\begin{array}{l}\text { Si a demanda: informar } \\
\text { para evitar duplicidad }\end{array}$ & $\begin{array}{l}\text { Si tratamiento instaurado: } \\
\text { informar para eliminar } \\
\text { duplicidad }\end{array}$ \\
\hline & & $\begin{array}{l}\text { Conservación } \\
\text { inadecuada }\end{array}$ & Dar pautas de conservación & \\
\hline \multicolumn{2}{|c|}{ Otros (indicar) } & & & \\
\hline
\end{tabular}

SPD: Sistema Personalizado de Dosificación. 


\section{Seguimiento Farmacoterapéutico}

En el caso de que durante la revisión de la medicación el farmacéutico detectara que el paciente presentaba posibles RNM, el farmacéutico podría llevar a cabo el servicio de SFT.

Siguiendo el Método Dáder de SFT (12), el farmacéutico podría realizar un estado de situación (emparejamiento de los problemas de salud con la medicación utilizada para su tratamiento), evaluaba si alguno de los problemas de salud era un RNM, lo clasificaba en necesidad, efectividad o seguridad y trataba de asociarlos con el PRM implicado. Realizaba el estudio del caso e intervenía con el paciente y/o médico para resolver los RNM y PRM detectados.

\section{Seguimiento del caso}

En función de la valoración del farmacéutico responsable, existía la posibilidad de realizar visitas sucesivas domiciliarias de seguimiento.

\section{Grado de satisfacción de los pacientes con el programa}

A finales de 2017, Hurkoa evaluó el grado de satisfacción de las personas incluidas en el programa de fragilidad, con el objetivo de conocer su percepción y plantear la finalización o extensión del mismo.

Se realizaron entrevistas a las personas atendidas, en las que se les preguntaba sobre el cumplimiento de expectativas, predisposición para seguir con el proyecto, valoración de la atención general recibida, valoración de la labor de profesionales de Hurkoa y valoración de la labor de la persona voluntaria de Hurkoa. Las respuestas a las cuestiones eran cerradas con 4 opciones de respuesta: [Muy alto / Alto / Normal / Bajo o Muy Bajo] o [Total / Bastante / A medias / Nada].

\section{RESULTADOS}

El Plan Integral de Atención a la Fragilidad de Hurkoa incluyó 15 usuarios en el municipio de Irún (edad: $80 \pm 2$ años) y 10 en el municipio de Azkoitia (edad: $80 \pm 3$ años). Tras la valoración de fragilidad por parte del técnico de Hurkoa, se ofreció el servicio de AFD a 12 pacientes (48\%). Un total de 6 pacientes (50\%), aceptaron recibir el servicio por parte del farmacéutico comunitario, 4 pacientes en el municipio de Irún atendidos por 3 farmacias y 2 en el municipio de Azkoitia, atendidos por 2 farmacias.

Las características de los pacientes incluidos en el programa se presentan en la tabla 2 . La edad media de los pacientes fue de $78 \pm 2$ años, con una distribución de género de 3 hombres y 3 mujeres. Todos los pacientes eran
Tabla 2 Características de los pacientes incluidos

\begin{tabular}{|c|c|l|c|}
\hline Paciente & Edad & Género & $\begin{array}{l}\text { No de medicamentos en } \\
\text { hoja de tratamiento activo }\end{array}$ \\
\hline 1 & 75 & Mujer & 13 \\
\hline 2 & 79 & Hombre & 7 \\
\hline 3 & 76 & Hombre & 8 \\
\hline 4 & 79 & Mujer & 16 \\
\hline 5 & 79 & Hombre & 6 \\
\hline 6 & 78 & Mujer & 8 \\
\hline
\end{tabular}

polimedicados con una media de 9 medicamentos por paciente.

La revisión del botiquín domiciliario por parte del farmacéutico detectó medicamentos caducados o no utilizados en todos los pacientes (tabla 3).

El farmacéutico retiró entre 2 y 3 medicamentos del botiquín a todos los pacientes, lo que implica aproximadamente el $15 \%$ del total de la medicación que tenían los pacientes en el domicilio (tabla 3 ). Una vez retirados los medicamentos, en los botiquines había entre 1 y 2 medicamentos más que los prescritos en la hoja de tratamiento activo. Esto se debía a que los pacientes tenían tratamientos antihistamínicos, antiinflamatorios, antipiréticos, etc. para patologias agudas puntuales.

En función de las necesidades del paciente, el farmacéutico realizó 1 visita a domicilio en 4 pacientes y 2 visitas en los 2 restantes.

La revisión de la medicación realizada por el farmacéutico detectó al menos un PRM en todos los pacientes del estudio (tabla 3). Un total de 3 pacientes (pacientes 2, 4, y 6) presentaban falta de adherencia involuntaria, por lo que en todos ellos el farmacéutico indicó la gestión de la medicación mediante SPD.

En el paciente 1 que presentaba, además de los problemas de salud crónicos, recidivas constantes de neumonía con ingresos hospitalarios frecuentes, el farmacéutico detectó una utilización incorrecta de Seretide ${ }^{\circledR}$ 25/250. La intervención del farmacéutico consistió en explicar la administración correcta de este inhalador y, en colaboración con el médico de atención primaria, se reforzó la pauta y modo de administración de Spiriva ${ }^{\circledR}$ y Ventolin ${ }^{\circledR}$, con la consiguiente remisión de las neumonías del paciente.

En el paciente 2, aparte de la falta de adherencia involuntaria como sucede en el paciente 4 y 6 , el farmacéutico detectó una importante interacción medicamentosa entre el 
Tabla 3 Datos del Servicio de AFD ofrecido a todos los pacientes

\begin{tabular}{|c|c|c|c|c|c|c|}
\hline \multirow[b]{2}{*}{ Paciente } & \multirow[b]{2}{*}{$\begin{array}{l}\mathrm{N}^{\circ} \text { de } \\
\text { visitas a } \\
\text { domicilio }\end{array}$} & \multicolumn{2}{|c|}{ Revisión del botiquín } & \multicolumn{3}{|c|}{ Revisión de la medicación } \\
\hline & & $\begin{array}{l}\text { No de } \\
\text { medicamentos } \\
\text { en botiquín }\end{array}$ & $\begin{array}{l}\mathrm{N}^{\circ} \text { de } \\
\text { medicamentos } \\
\text { retirados }\end{array}$ & PRM & $\begin{array}{l}\text { Medicamento } \\
\text { involucrado }\end{array}$ & Intervención del farmacéutico \\
\hline 1 & 2 & 17 & 3 & $\begin{array}{l}\text { Administración } \\
\text { errónea }\end{array}$ & $\begin{array}{l}\text { Seretide }{ }^{\circledR} \\
25 / 250\end{array}$ & $\begin{array}{l}\text { Educación en el uso de } \\
\text { inhaladores. }\end{array}$ \\
\hline 2 & 1 & 10 & 3 & $\begin{array}{l}\text { Falta de } \\
\text { adherencia } \\
\text { (involuntaria) } \\
\text { Interacción }\end{array}$ & $\begin{array}{l}\text { Plenur }^{\circledR} \\
\text { Elontril }^{\circledR} \\
\text { Plenur }_{+}+ \\
\text {Exforge }^{\circledR}\end{array}$ & $\begin{array}{l}\text { Realización de SPD y } \\
\text { supervisión periódica. } \\
\text { Vigilancia de la aparición de } \\
\text { signos de toxicidad. }\end{array}$ \\
\hline 3 & 1 & 12 & 2 & $\begin{array}{l}\text { Probabilidad } \\
\text { de aparición de } \\
\text { efectos adversos }\end{array}$ & $\begin{array}{l}\text { Akineton }{ }^{\circledR} \\
\text { Zeldox }^{\circledR}\end{array}$ & $\begin{array}{l}\text { Vigilancia de la aparición de } \\
\text { signos y RAM. Explicación } \\
\text { para el conocimiento de la } \\
\text { medicación. } \\
\text { Consejos higiénico-dietéticos. }\end{array}$ \\
\hline 4 & 1 & 18 & 2 & $\begin{array}{l}\text { Falta de } \\
\text { adherencia } \\
\text { (involuntaria) }\end{array}$ & & $\begin{array}{l}\text { Realización de SPD y } \\
\text { supervisión periódica. }\end{array}$ \\
\hline 5 & 2 & 10 & 2 & $\begin{array}{l}\text { Falta de } \\
\text { adherencia } \\
\text { (voluntaria) }\end{array}$ & Donepezilo ${ }^{\circledR}$ & $\begin{array}{l}\text { Comunicación con el médico. } \\
\text { Eliminar miedo a los efectos } \\
\text { adversos, explicar importancia } \\
\text { de la medicación para el } \\
\text { problema de salud, etc. }\end{array}$ \\
\hline 6 & 1 & 12 & 3 & $\begin{array}{l}\text { Falta de } \\
\text { adherencia } \\
\text { (involuntaria) }\end{array}$ & & $\begin{array}{l}\text { Realización de SPD y } \\
\text { supervisión periódica. }\end{array}$ \\
\hline
\end{tabular}

RAM: Reacción Adversa Medicamentosa. SPD: Sistema Personalizado de Dosificación. PRM: Problema Relacionado con la Medicación.

Plenur ${ }^{\circledR}$ y el Exforge ${ }^{\circledR}$, por lo que se controlaron los signos indicativos de toxicidad de litio como diarrea, vómitos, cansancio, debilidad muscular, falta de coordinación, vértigo, visión borrosa, poliuria, entre otros.

En el paciente 3 el farmacéutico detectó una importante probabilidad de aparición de efectos adversos relacionados principalmente con dos de los tratamientos prescritos (Akineton ${ }^{\circledR}$ y Zeldox ${ }^{\circledR}$ ), por lo que intervino con el paciente, reforzando el conocimiento ofreciéndole información personalizada sobre su medicación y educándole en el uso correcto de la medicación prescrita, así como realizando una vigilancia estrecha del control del pulso cardíaco y ofreciendo consejos higiénico-dietéticos.

El paciente 5 presentó falta de adherencia voluntaria al tratamiento con Donepezilo ${ }^{\circledR}$, ya que refería que le sentaba mal y que le provocaba diarreas y mareos, entre otras reacciones. El farmacéutico intervino para reforzar la importancia de la adherencia al tratamiento, eliminado los miedos que el paciente refería, con recomendaciones higiénico-dietéticas y explicando la importancia de tomar el medicamento para el control de su enfermedad.

En la evaluación de la satisfacción en el Plan Integral de Atención a la Fragilidad de Hurkoa que incluía la AFD (figura 2), el $100 \%$ de los pacientes $(n=6)$ refirieron un grado de satisfacción alto o muy alto con el programa, así como una total conformidad con la necesidad de implementar un programa de estas características (figura 2).

Los buenos resultados obtenidos han impulsado al COFG y a Hurkoa a extender el programa por la provincia de Gipuzkoa y en la actualidad se está iniciando la implantación del programa en dos nuevos municipios (Pasaia y Arrasate). 


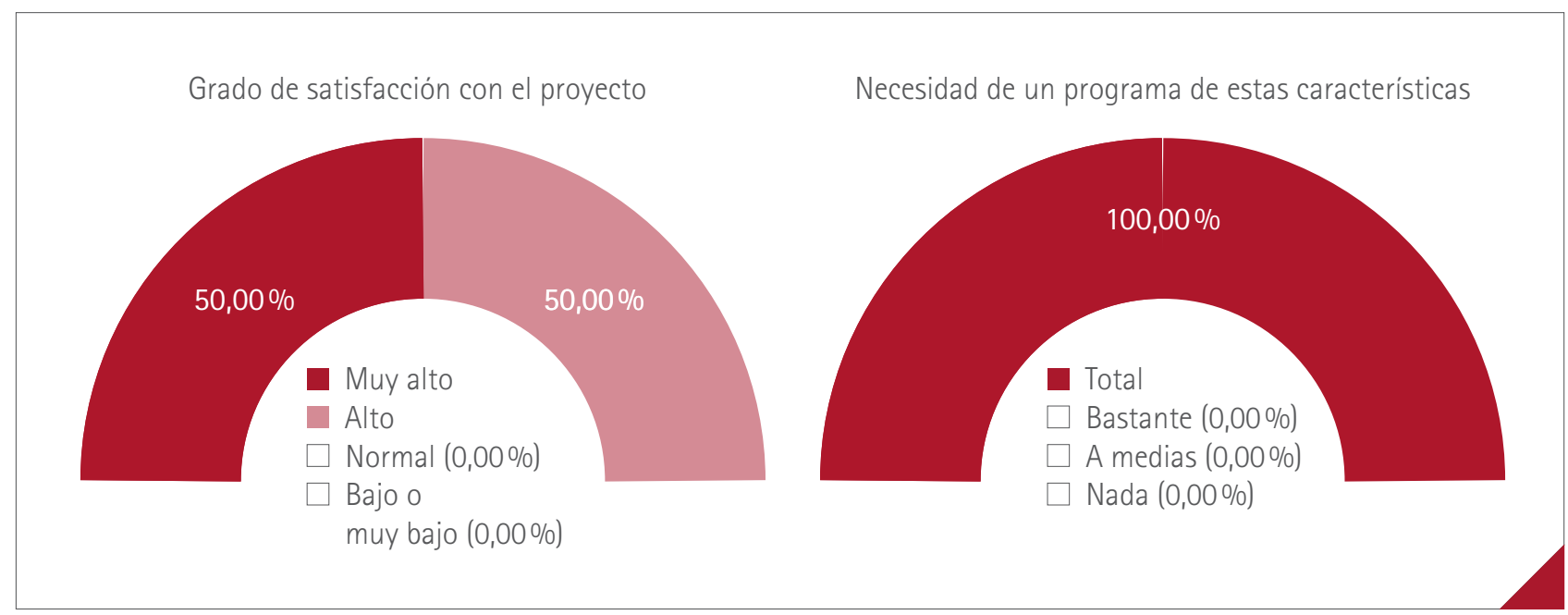

Figura 2 Grado de satisfacción de los usuarios incluidos en el Programa de Fragilidad de Hurkoa

\section{DISCUSIÓN}

El presente programa piloto ha demostrado que el farmacéutico comunitario es un profesional clave que debe estar integrado en una atención domiciliaria multidisciplinar.

La coordinación entre los distintos profesionales sanitarios (personal de medicina, personal de farmacia, personal de enfermería, etc.) y entre distintos niveles asistenciales facilita, entre otros, la educación sanitaria de los ciudadanos, la gestión de patologías crónicas, la detección proactiva de posibles problemas relacionados con el uso de los medicamentos y la prevención de enfermedades; hecho relevante en la atención domiciliaria de las personas dependientes y vulnerables.

La experiencia llevada a cabo en nuestro proyecto ha puesto en relieve el gran número de actuaciones e intervenciones realizadas por el farmacéutico. Entre ellas, cabe destacar que se han retirado medicamentos y se han detectado PRM en todos los pacientes.

Respecto a la retirada de medicamentos, el farmacéutico intervino en todos los pacientes con una retirada mínima de 2 medicamentos por paciente. Algunos, se retiraron por la caducidad y mala conservación, aunque un elevado porcentaje eran tratamientos antibióticos sin acabar. La resistencia a los antibióticos es hoy en día una de las mayores amenazas para la salud mundial (13), por lo que la intervención contribuyó en abordar la problemática de la automedicación y así hacer frente a dicha problemática.

La falta de adherencia terapéutica fue el PRM más identificado por parte del farmacéutico comunitario. De hecho, la mitad de los pacientes presentaron falta de adherencia involuntaria. Este problema de falta de adherencia al tratamiento es un problema frecuente a nivel mundial y se estima que el $50 \%$ de los medicamentos crónicos no se administran de forma adecuada. En pacientes de edad avanzada el incumplimiento involuntario es una causa frecuente de falta de adherencia debido al olvido (14).
En nuestro estudio, la intervención más utilizada en estos pacientes, debido a la eficacia demostrada (15), al grado de dependencia de los pacientes atendidos y la complejidad de los tratamientos, ha sido el uso de SPD.

Además de las intervenciones, todos los pacientes incluidos en el programa piloto refirieron un grado de satisfacción alto con el programa, al igual que confirmaron la necesidad de implementar un programa de dichas características. Por ello, la inclusión del farmacéutico comunitario en el equipo multidisciplinar y la importancia de seguir trabajando en el programa no es solo una conclusión de los resultados, sino también, una realidad expuesta por los propios pacientes.

La AFD es un servicio que pretende dar respuesta a una necesidad identificada hace tiempo que, hay que insistir, no consiste únicamente en la dispensación de medicamentos al domicilio $(5,16)$.

A nivel internacional, existen experiencias de AFD. Hay ciertos programas que se han realizado en países como Australia (Meds Check: Medicines Use Review y Home Medicines Review), Canadá (Meds Check at home) y Reino Unido (Medicines AssesmentCCompliance Support) (6-9) en los que se ha podido observar, al igual que en nuestro programa piloto, que los farmacéuticos identificaron y resolvieron gran cantidad de PRM tras la asistencia del farmacéutico en el domicilio. Programas de este calibre ayudan a los pacientes en la correcta compresión del uso de los medicamentos al igual que en la obtención de conocimientos higiénico-dietéticos y en la facilitación de información al paciente sobre el correcto almacenamiento de determinados fármacos que requieren condiciones especiales, consiguiendo así mejoras importantes en su calidad de vida.

Varias publicaciones han señalado al propio domicilio como lugar de origen de los errores de medicación y resaltado el papel del farmacéutico comunitario en la detección y prevención de estos errores desde la farmacia (17-19). 
En cuanto a las limitaciones del estudio, hay que destacar que a pesar de que el tamaño muestral sea limitado, es una buena aproximación para demostrar la necesidad de la inclusión de la figura del farmacéutico asistencial en el domicilio.

En cuanto a la labor del farmacéutico, conviene resaltar que la participación fue voluntaria y no percibieron remuneración alguna.

La colaboración entre dos entidades tan diferentes como Hurkoa y COFG se considera un éxito, ya que todos los pacientes que han recibido los servicios se han visto beneficiados por el mismo. Colaboraciones de este alcance nos permiten complementar lo social con lo sanitario, creando sinergias e impulsando proyectos sociosanitarios con resultados muy positivos.

Parece interesante ampliar este servicio a sectores más amplios de la población con esta necesidad. Por todo ello, y tras los resultados obtenidos en este programa piloto, desde el COFG se pretende reforzar la colaboración con Hurkoa con su ampliación a otros municipios e impulsar la atención farmacéutica de los pacientes frágiles en el propio domicilio del paciente con la reciente creación de la nueva comisión de AFD del COFG (20).

El presente estudio refuerza la importancia de la inclusión del farmacéutico comunitario en el equipo multidisciplinar de AFD, ofreciendo respuestas individualizadas a cada paciente, mejorando la gestión de su medicación y así consiguiendo mejoras significativas en la calidad de vida $y_{\text {, }}$ en definitiva, contribuyendo a la sostenibilidad del sistema sanitario.

\section{AGRADECIMIENTOS}

A la Fundación Hurkoa por su colaboración en el proyecto.

\section{REFERENCIAS BIBLIOGRÁFICAS}

1. Organización Mundial de la Salud (OMS). Informe mundial sobre el envejecimiento y la salud. 2015. Disponible en: https://apps.who.int/ iris/bitstream/handle/10665/186466/9789240694873_spa.pdf?sequence $=1$

2. Sociedad Española de Farmacia Familiar y comunitaria. Propuesta del papel del Farmacéutico comunitario en la Atención sociosanitaria. 2016. Disponible en: http://www.newhealthfoundation.org/web/ wp-content/uploads/2017/01/PROPUESTA-SEFAC-ATENCION-SOCIOSANITARIA.pdf

3. Ministerio de Sanidad, Consumo y Bienestar Social. Informe de evaIuación Estrategia Cronicidad. 2019. Disponible en: https://www. mscbs.gob.es/organizacion/sns/planCalidadSNS/pdf/Evaluacion_E._ Cronicidad_Final.pdf
4. Consejo de Europa. Comité de Ministros. Resolución ResAP relativa al papel del farmacéutico en el marco de la seguridad de la salud. Pharm Care Esp. 2001; 3(3): 216-222.

5. Consejo General de Colegios Farmacéuticos. Atención Farmacéutica Domiciliaria. 2006. Disponible en: https://www.portalfarma.com/inicio/serviciosprofesionales/pacientedependiente/Documents/at_farma_domiciliaria.pdf

6. Miller E, Emblen G. Home medicines review: the how and why for GPs. Australian Family Physician. ISSN: 0300-8495; 2004 Jan;33.

7. White L. Consumer perspectives of the Australian Home Medicines Review Program: Benefits and barriers. Research in Social \&t Administrative Pharmacy. Australia; 2011 Disponible en: http://www.rsap. org/article/S1551-7411(10)00166-X/abstract

8. Ontario G of, Health M of, Care L-T. MedsCheck at Home - Health Care Professionals - MOHLTC. Government of Ontario, Ministry of Health and Long-Term Care; 2013 Disponible en: http://www.health.gov.on.ca/en/pro/programs/drugs/medscheck/medscheck_home. aspx

9. Jellyhaus. EN6 Medicines Assessment \& Compliance Support. Pharmaceutical Services Negotiating Committee. London: PSNC Main site. Disponible en: http://psnc.org.uk/services-commissioning/locally-commissioned-services/en6-medicines-assessment-compliance-support/

10. El Global. Farmacia [Internet] Domi-EQuifar: La AF domiciliaria da resultados en Comunidad Valenciana. 08/02/2019. Disponible en: https://elglobal.es/farmacia/domi-eqifar-la-af-domiciliaria-da-resultados-en-comunidad-valenciana-ml1915394/

11. Departamento de Empleo y Políticas Sociales. Gobierno Vasco. Informe del proyecto de fragilidad. 2018. Disponible en: https:// www.hurkoa.eus/sites/default/files/memorias/INFORME_FRAGILIDAD2018_ES_WEB.pdf

12. Sabater, D. Silva, MM., Faus MJ. Método Dáder: Guía de Seguimiento Farmacoterapéutico. 3a Edición. ISBN: 978-84-608-0604-2. Disponible en: http://www.ugr.es/ cts131/esp/guias/GUIA\%20FINAL\%20 DADER.pdf

13. Organización Mundial de la Salud (OMS). Resistencia a los antibióticos. 2020. Disponible en: https://www.who.int/es/news-room/factsheets/detail/resistencia-a-los-antibi\%C3\%B3ticos

14. Organización Mundial de la Salud (OMS). Adherence to long-term therapies. Evidence for action. 2003. Disponible en: https://www. who.int/chp/knowledge/publications/adherence_report/en/

15. FUNDAMED. Cronicidad, Adherencia y Sistemas Personalizados de Dosificación (SPD). 2017. Disponible en: https://www.sefac.org/sites/ default/files/2018-04/TT\%20Cronicidad\%2C\%20adherencia\%20 y\%20SPD_Esp.pdf

16. Ministerio de Sanidad y Consumo. Consenso sobre Atención Farmacéutica. Madrid; 2001. Disponible en: https://www.mscbs.gob.es/ profesionales/farmacia/consenso/home.htm

17. Casas P, Ojeda M, González M, Rabasco A. Implementación de herramientas informáticas en la detección y prevención de errores de medicación desde la farmacia comunitaria. Rev. O.F.I.L. 2015; 25; 3:165-173.

18. Salar L, Velert J, Climent MT, Escutia M, Aznar S, Garcia-Cebrián F. Capacidad de la oficina de farmacia de detectar y corregir automedicación incorrecta. Pharm Care Esp. 2005; 7(Extr):78.

19. Garcia de Bikuña B, Del Arco J, Seisdedos N. Las especialidades farmacéuticas complejas: Información al paciente sobre su manejo. Argibideak 2002; 12(1): 1-4.

20. Botika XXI. Nueva Comisión de Atención Farmacéutica Domiciliaria del Colegio. 2020. №49. Pág.5. 